\title{
New exponential stability criteria for neutral system with time-varying delay and nonlinear perturbations
}

\author{
Yuechao Ma and Lihong Zhu*
}

"Correspondence:

lihongzhu88@163.com

College of Science, Yanshan

University, 438 Hebeidajie Road,

Haigang District, Qinhuangdao,

Hebei 066004, P.R. China

\begin{abstract}
In this paper, the problem of exponential stability for neutral system with time-varying delay and nonlinear perturbations is investigated. By using the technology of model transformations, based on a linear matrix inequality (LMI) and a generalized Lyapunov-Krasovskii function, a new criterion for exponential stability with delay dependence is obtained. Due to a new integral inequality, the result is less conservative. Finally, some numerical examples are presented to illustrate the effectiveness of the method.
\end{abstract}

Keywords: neutral system; nonlinear perturbations; exponential stability; linear matrix inequality

\section{Introduction}

Time delay is frequently viewed as a source of instability, and it is encountered in various engineering systems such as electrical circuits, chemical processes, networked control systems power systems, and other areas [1,2]. Current efforts on the problem of the stability of time-delay systems can be divided into two categories, namely delay-independent criteria and delay-dependent criteria. A number of delay-independent sufficient conditions for the asymptotic stability of delay systems have been presented by various researchers (for example [3]). Also a few delay-dependent sufficient conditions have been shown in [4, 5]. So the problem of robust stability analysis for time-delay neutral systems is important both in theory and in practice and is of interest to many researchers; see [6,7] and the references therein.

Recently, many researchers have paid a lot of attention to the problem of robust stability for delay systems with nonlinear uncertainties [2, 8-11], and many methods have been proposed to deal with nonlinear uncertainties (see for example [10,11]). For instance, by using a descriptor model transformation and decomposition technique, some delay-dependent stability criteria are obtained in [2]. In [10] the stability conditions are developed by a descriptor model transformation technique, and the nonlinear uncertainties are handled by the S-procedure. However, these results are only concerned with asymptotic stability, without providing any conditions for exponential stability and any information as regards the decay rates.

As is well known, exponential stability converges faster than others, so the issue of exponential stability for some systems with time delay has received considerable attention

o2014 Ma and Zhu; licensee Springer. This is an Open Access article distributed under the terms of the Creative Commons Attribution License (http://creativecommons.org/licenses/by/2.0), which permits unrestricted use, distribution, and reproduction in any medium, provided the original work is properly cited. 
in recent years [11-14]. For example, Liu [12] has investigated the exponential stability of a general power system. Kwon and Park discussed the exponential stability of uncertain dynamic systems including state delay in [13].

Considering those, many researchers have studied the exponential stability analysis for neutral systems with time-varying and nonlinear perturbations [15-18]. Chen et al. [15] presented a new criterion for exponential stability for uncertain neutral systems with nonlinear perturbations by employing an integral inequality. Ali [16] investigated the exponential stability for a neutral delay differential system with nonlinear uncertainties by following a generalized eigenvalue problem approach.

Based on the above, the exponential stability of neutral system with nonlinear uncertainties is discussed in this paper, and by employing a Lyapunov-Krasovskii function, the LMI method, and a new integral inequality, a sufficient condition for exponential stability of the system is provided. Finally, some numerical examples are presented to illustrate the effectiveness of the method.

\section{System description and preliminary lemma}

Consider the neutral system with delay and nonlinear uncertainties described by

$$
\left\{\begin{aligned}
\dot{x}(t)= & A x(t)+B x(t-d(t))+C \dot{x}(t-d(t)) \\
& +G_{1} f_{1}(t, x(t))+G_{2} f_{2}(t, \dot{x}(t))+G_{3} f_{3}(t, x(t-d(t)))+G_{4} f_{4}(t, \dot{x}(t-d(t))), \\
x(t)= & \phi(t), \quad \dot{x}(t)=\varphi(t), \quad t \in\left[-d_{2}, 0\right]
\end{aligned}\right.
$$

where $x(t) \in R^{n}$ is the state vector, and $A, B, C, G_{1}, G_{2}, G_{3}$, and $G_{4} \in R^{n \times n}$ are known real parameter matrices of appropriate dimensions. $d(t)$ is for the time-varying continuous functions satisfying $d_{1} \leq d(t) \leq d_{2}, 0 \leq \dot{d}(t) \leq \bar{d}$, in which $d, d_{1}, d_{2}$ and $\bar{d}$ are positive constants. $\phi(\cdot)$ and $\varphi(\cdot) \in L_{2}\left[-d_{2}, 0\right]$ are given continuous vector-valued initial functions. $f_{1}(t, x(t)), f_{2}(t, x(t-d(t))), f_{3}(t, \dot{x}(t))$ and $f_{4}(t, \dot{x}(t-d(t)))$ are nonlinear uncertainties and satisfy the following conditions:

$$
\begin{aligned}
& \left\|f_{1}(t, x(t))\right\| \leq \alpha_{1}\|x(t)\|, \\
& \left\|f_{2}(t, \dot{x}(t))\right\| \leq \alpha_{2}\|\dot{x}(t)\|, \\
& \left\|f_{3}(t, x(t-d(t)))\right\| \leq \alpha_{3}\|x(t-d(t))\|, \\
& \left\|f_{4}(t, \dot{x}(t-d(t)))\right\| \leq \alpha_{4}\|\dot{x}(t-d(t))\|, \quad t>0 .
\end{aligned}
$$

Let $\dot{x}(t)=y(t)$, then system (1) is equivalent to the following:

$$
\left\{\begin{aligned}
y(t)= & A x(t)+B x(t-d(t))+C y(t-d(t)) \\
& +G_{1} f_{1}(t, x(t))+G_{2} f_{2}(t, y(t))+G_{3} f_{3}(t, x(t-d(t)))+G_{4} f_{4}(t, y(t-d(t))), \\
\dot{x}(t)= & y(t), \quad t \in\left[-d_{2}, 0\right] .
\end{aligned}\right.
$$

Definition 1 Consider the linear system (2). If there exist two scalars $\varepsilon>0$ and $\gamma \geq 1$ such that

$$
\|\mu(t)\| \leq \gamma e^{-\varepsilon t}\|\Phi\|_{d}, \quad t \geq 0,
$$


and we let $\|\Phi\|_{d}=\sup _{-d \leq s \leq 0} \sqrt{\|\mu(s)\|^{2}+\|\dot{\mu}(s)\|^{2}}$, then system (2) is exponentially stable, where $\varepsilon$ is called the exponential convergence rate.

Before proceeding with the main results, several lemmas are necessary.

Lemma 1 For any symmetric positive-definite constant matrix $R=\left[\begin{array}{ll}R_{1} & R_{2} \\ * & R_{3}\end{array}\right]>0$, where $R_{1}, R_{2}, R_{3} \in R^{n \times n}$, and $0<h(t) \leq h$, if there exists a vector function $\dot{x}(\cdot):[0, h] \rightarrow R^{n}$ such that the following integration is well defined, then we have

$$
\begin{aligned}
-h(t) & \int_{t-h(t)}^{t} \eta^{T}(s) R \eta(s) d s \\
\leq & {\left.\left[\begin{array}{c}
x(t) \\
x(t-h(t)) \\
\int_{t-h(t)}^{t} x(s) d s
\end{array}\right]\right]^{T}\left[\begin{array}{ccc}
-R_{3} & R_{3} & -R_{2}^{T} \\
* & -R_{3} & R_{2} \\
* & * & -R_{1}
\end{array}\right]\left[\begin{array}{c}
x(t) \\
x(t-h(t)) \\
\int_{t-h(t)}^{t} x(s) d s
\end{array}\right], }
\end{aligned}
$$

where $\eta(t)=\left(x^{T}(t) \dot{x}^{T}(t)\right)^{T}$.

Proof

$$
\begin{aligned}
-h(t) & \int_{t-h(t)}^{t} \eta^{T}(s) R \eta(s) d s \\
\leq & -\int_{t-h(t)}^{t}\left[\begin{array}{c}
x(s) \\
\dot{x}(s)
\end{array}\right]^{T} d s\left[\begin{array}{cc}
R_{1} & R_{2} \\
* & R_{3}
\end{array}\right] \int_{t-h(t)}^{t}\left[\begin{array}{l}
x(s) \\
\dot{x}(s)
\end{array}\right] d s \\
& =\left[\begin{array}{c}
x(t) \\
x(t-h(t)) \\
\int_{t-h(t)}^{t} x(s) d s
\end{array}\right]^{T}\left[\begin{array}{ccc}
-R_{3} & R_{3} & -R_{2}^{T} \\
* & -R_{3} & R_{2} \\
* & * & -R_{1}
\end{array}\right]\left[\begin{array}{c}
x(t) \\
x(t-h(t)) \\
\int_{t-h(t)}^{t} x(s) d s
\end{array}\right] .
\end{aligned}
$$

Remark 1 Lemma 1 will play a key role in the derivation of a less conservative delaydependent condition.

Lemma 2 [19] Let $U, V, W$, and $M$ be real matrices of appropriate dimensions with $M$ satisfying $M=M^{T}>0$, then

$$
M+U V W+W^{T} V^{T} U^{T}<0
$$

for all $V V^{T} \leq I$, if and only if there exists a scalar $\varepsilon>0$ such that

$$
M+\varepsilon^{-1} U U^{T}+\varepsilon W^{T} W<0 .
$$

\section{Main results}

Theorem 1 For the prescribed scalar $d_{2}>0$, system (2) is globally exponentially stable with a convergence rate, if there exist positive-definite matrices $P=\left[\begin{array}{ll}P_{1} & 0 \\ P_{2} & P_{3}\end{array}\right], P_{1}^{T}=P_{1}, P_{3}^{T}=P_{3}$, for positive-definite matrices $Q_{1}, Q_{2}, Q_{3}, W=\left[\begin{array}{cc}W_{1} & W_{2} \\ * & W_{3}\end{array}\right]$ and real matrix $Z=\left[\begin{array}{cc}Z_{1} & Z_{2} \\ * & Z_{3}\end{array}\right]$ satisfying 
the following LMI:

$$
\begin{aligned}
& \Pi=\left[\begin{array}{cccccccccccc}
\Pi_{11} & \Pi_{12} & 0 & \Pi_{14} & P_{2}^{T} B & P_{2}^{T} C & P_{2}^{T} G_{1} & P_{2}^{T} G_{2} & P_{2}^{T} G_{3} & P_{2}^{T} G_{4} & \Pi_{1,11} & 0 \\
* & \Pi_{22} & 0 & 0 & P_{3} B & P_{3} C & P_{3} G_{1} & P_{3} G_{2} & P_{3} G_{3} & P_{3} G_{4} & 0 & 0 \\
* & * & \Pi_{33} & \Pi_{34} & 0 & 0 & 0 & 0 & 0 & 0 & 0 & \Pi_{3,12} \\
* & * & * & \Pi_{44} & 0 & 0 & 0 & 0 & 0 & 0 & \Pi_{4,11} & \Pi_{4,12} \\
* & * & * & * & \Pi_{55} & 0 & 0 & 0 & 0 & 0 & 0 & 0 \\
* & * & * & * & * & \Pi_{66} & 0 & 0 & 0 & 0 & 0 & 0 \\
* & * & * & * & * & * & -\varepsilon_{1} I & 0 & 0 & 0 & 0 & 0 \\
* & * & * & * & * & * & * & -\varepsilon_{2} I & 0 & 0 & 0 & 0 \\
* & * & * & * & * & * & * & * & -\varepsilon_{3} I & 0 & 0 & 0 \\
* & * & * & * & * & * & * & * & * & -\varepsilon_{4} I & 0 & 0 \\
* & * & * & * & * & * & * & * & * & * & \Pi_{11,11} & 0 \\
* & * & * & * & * & * & * & * & * & * & * & \Pi_{12,12}
\end{array}\right] \\
&<0
\end{aligned}
$$

where

$$
\begin{aligned}
& \Pi_{11}=P_{2}^{T} A+A^{T} P_{2}+Q_{1}+Q_{2}+Q_{3}+d_{2} W_{1}+\left(d_{2}-d_{1}\right) Z_{1}-\frac{1}{d_{2}} W_{3}+\beta_{1}^{2} \varepsilon_{1} I, \\
& \Pi_{12}=P_{1}-P_{2}^{T}+A^{T} P_{3}+d_{2} W_{2}+\left(d_{2}-d_{1}\right) Z_{2}, \quad \Pi_{14}=-\frac{1}{d_{2}} W_{3}, \\
& \Pi_{22}=2 P_{3}+d_{2} W_{3}+\left(d_{2}-d_{1}\right) Z_{3}+R+\beta_{2}^{2} \varepsilon_{2} I, \quad \Pi_{1,11}=-\frac{1}{d_{2}} W_{2}^{T}, \\
& \Pi_{33}=-Q_{1}-\frac{1}{d_{2}-d_{1}} Z_{3}, \quad \Pi_{34}=\frac{1}{d_{2}-d_{1}} Z_{3}, \quad \Pi_{3,12}=-\frac{1}{d_{2}-d_{1}} Z_{2}^{T}, \\
& \Pi_{44}=-Q_{2}-\frac{1}{d_{2}-d_{1}} Z_{3}-\frac{1}{d_{2}} W_{3}, \quad \Pi_{4,11}=\frac{1}{d_{2}} W_{2}^{T}, \quad \Pi_{4,12}=\frac{1}{d_{2}-d_{1}} Z_{2}^{T}, \\
& \Pi_{55}=-Q_{3}+\beta_{3}^{2} \varepsilon_{3} I, \quad \Pi_{66}=-(1-\bar{d}) R+\beta_{4}^{2} \varepsilon_{4} I, \\
& \Pi_{11,11}=-\frac{1}{d_{2}} W_{1}, \quad \Pi_{12,12}=-\frac{1}{d_{2}-d_{1}} Z_{1} .
\end{aligned}
$$

Proof Let us consider the Lyapunov functional candidate to be

$$
V(t)=V_{1}(t)+V_{2}(t)+V_{3}(t)+V_{4}(t)+V_{5}(t)+V_{6}(t),
$$

where

$$
\begin{aligned}
& V_{1}(t)=\left[\begin{array}{ll}
x^{T}(t) & y^{T}(t)
\end{array}\right]\left[\begin{array}{ll}
I & 0 \\
0 & 0
\end{array}\right]\left[\begin{array}{cc}
P_{1} & 0 \\
P_{2} & P_{3}
\end{array}\right]\left[\begin{array}{l}
x(t) \\
y(t)
\end{array}\right], \\
& V_{2}(t)=\int_{t-d_{1}}^{t} x^{T}(s) Q_{1} x(s) d s, \quad V_{3}(t)=\int_{t-d_{2}}^{t} x^{T}(s) Q_{2} x(s) d s, \\
& V_{4}(t)=\int_{-d_{2}}^{0} \int_{t+\beta}^{t} \eta^{T}(s) W \eta(s) d s d \beta+\int_{-d_{2}}^{-d_{1}} \int_{t+\beta}^{t} \eta^{T}(s) Z \eta(s) d s d \beta, \\
& V_{5}(t)=\int_{-d_{2}}^{0} F(t, s) d s, \quad \text { with } F(t, s)=\sup _{s \leq \xi \leq 0} x^{T}(t+\xi) Q_{3} x(t+\xi),
\end{aligned}
$$

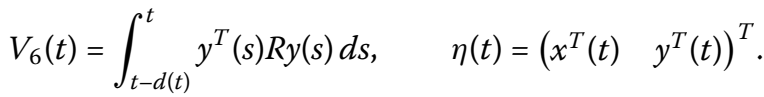


Then the time derivative of $V\left(x_{t}\right)$ along the solution of system (1) gives

$$
\begin{aligned}
\dot{V}(t)= & \dot{V}_{1}(t)+\dot{V}_{2}(t)+\dot{V}_{3}(t)+\dot{V}_{4}(t)+\dot{V}_{5}(t)+\dot{V}_{6}(t), \\
\dot{V}_{1}(t)= & 2\left[\begin{array}{ll}
x^{T}(t) & y^{T}(t)
\end{array}\right]\left[\begin{array}{cc}
P_{1} & P_{2}^{T} \\
0 & P_{3}
\end{array}\right]\left[\begin{array}{c}
\dot{x}(t) \\
0
\end{array}\right] \\
= & 2\left[\begin{array}{ll}
x^{T}(t) & \left.y^{T}(t)\right]
\end{array}\right]\left[\begin{array}{cc}
P_{1} & P_{2}^{T} \\
0 & P_{3}
\end{array}\right]\left[\begin{array}{c}
A x(t)+B x(t-d(t))-y(t) \\
+C y(t-d(t))+G_{1} f_{1}(t, x(t))+G_{2} f_{2}(t, y(t)) \\
+G_{3} f_{3}(t, x(t-d(t)))+G_{4} f_{4}(t, y(t-d(t)))
\end{array}\right], \\
\dot{V}_{2}(t)= & x^{T}(t) Q_{1} x(t)-x^{T}\left(t-d_{1}\right) Q_{1} x\left(t-d_{1}\right), \\
\dot{V}_{3}(t)= & x^{T}(t) Q_{2} x(t)-x^{T}\left(t-d_{2}\right) Q_{2} x\left(t-d_{2}\right), \\
\dot{V}_{4}(t)= & \int_{-d_{2}}^{0}\left[\begin{array}{l}
x(s) \\
y(s)
\end{array}\right]^{T}\left[\begin{array}{ll}
W_{1} & W_{2} \\
* & W_{3}
\end{array}\right]\left[\begin{array}{l}
x(s) \\
y(s)
\end{array}\right] d s-\int_{t-d_{2}}^{t}\left[\begin{array}{l}
x(s) \\
y(s)
\end{array}\right]^{T}\left[\begin{array}{cc}
W_{1} & W_{2} \\
* & W_{3}
\end{array}\right]\left[\begin{array}{l}
x(s) \\
y(s)
\end{array}\right] d s \\
& +\int_{-d_{2}}^{-d_{1}}\left[\begin{array}{l}
x(s) \\
y(s)
\end{array}\right]^{T}\left[\begin{array}{cc}
Z_{1} & Z_{2} \\
* & Z_{3}
\end{array}\right]\left[\begin{array}{l}
x(s) \\
y(s)
\end{array}\right] d s-\int_{t-d_{2}}^{t-d_{1}}\left[\begin{array}{l}
x(s) \\
y(s)
\end{array}\right]^{T}\left[\begin{array}{cc}
Z_{1} & Z_{2} \\
* & Z_{3}
\end{array}\right]\left[\begin{array}{l}
x(s) \\
y(s)
\end{array}\right] d s .
\end{aligned}
$$

By Lemma 1, we get

$$
\begin{aligned}
\dot{V}_{4}(t) \leq & h_{2}\left[\begin{array}{l}
x(t) \\
y(t)
\end{array}\right]^{T}\left[\begin{array}{ll}
W_{1} & W_{2} \\
* & W_{3}
\end{array}\right]\left[\begin{array}{l}
x(t) \\
y(t)
\end{array}\right]+\left(h_{2}-h_{1}\right)\left[\begin{array}{l}
x(t) \\
y(t)
\end{array}\right]^{T}\left[\begin{array}{cc}
Z_{1} & Z_{2} \\
* & Z_{3}
\end{array}\right]\left[\begin{array}{l}
x(t) \\
y(t)
\end{array}\right] \\
& +\frac{1}{h_{2}}\left[\begin{array}{c}
x(t) \\
x\left(t-h_{2}\right) \\
\int_{t-h_{2}}^{t} x(s) d s
\end{array}\right]^{T}\left[\begin{array}{ccc}
-W_{3} & W_{3} & -W_{2}^{T} \\
* & -W_{3} & W_{2} \\
* & * & -W_{1}
\end{array}\right]\left[\begin{array}{c}
x(t) \\
x\left(t-h_{2}\right) \\
\int_{t-h_{2}}^{t} x(s) d s
\end{array}\right] \\
& +\frac{1}{h_{2}-h_{1}}\left[\begin{array}{c}
x\left(t-h_{1}\right) \\
x\left(t-h_{2}\right) \\
\int_{t-h_{2}}^{t-h_{1}} x(s) d s
\end{array}\right]^{T}\left[\begin{array}{ccc}
-Z_{3} & Z_{3} & -Z_{2}^{T} \\
* & -Z_{3} & Z_{2} \\
* & * & -Z_{1}
\end{array}\right]\left[\begin{array}{c}
x\left(t-h_{1}\right) \\
x\left(t-h_{2}\right) \\
\int_{t-h_{2}}^{t-h_{1}} x(s) d s
\end{array}\right], \\
\dot{V}_{5}(t) \leq & x^{T}(t) Q_{3} x(t)-x^{T}(t-d(t)) Q_{3} x(t-d(t)), \\
\dot{V}_{6}(t) \leq & y^{T}(t) R y(t)-y^{T}(t-d(t)) R y(t-d(t))(1-\bar{d}) .
\end{aligned}
$$

Since

$$
\begin{aligned}
& \varepsilon_{1}\left[\beta_{1}^{2} x^{T}(t) x(t)-f_{1}^{T}(t, x(t)) f_{1}(t, x(t))\right] \geq 0, \\
& \varepsilon_{2}\left[\beta_{2}^{2} y^{T}(t) y(t)-f_{2}^{T}(t, y(t)) f_{2}(t, y(t))\right] \geq 0, \\
& \varepsilon_{3}\left[\beta_{3}^{2} x^{T}(t-d(t)) x(t-d(t))-f_{3}^{T}(t, x(t-d(t))) f_{3}(t, x(t-d(t)))\right] \geq 0, \\
& \varepsilon_{4}\left[\beta_{4}^{2} y^{T}(t-d(t)) y(t-d(t))-f_{4}^{T}(t, y(t-d(t))) f_{4}(t, y(t-d(t)))\right] \geq 0,
\end{aligned}
$$

by Lemma 2, we get

$$
-h \int_{t-h(t)}^{t} \dot{x}^{T}(s) W \dot{x}(s) d s \leq z(t)^{T}\left[\begin{array}{cc}
-W & W \\
* & -W
\end{array}\right] z(t),
$$

where $z(t)=\left[x^{T}(t) x^{T}(t-h(t))\right]^{T}$. 
So

$$
\begin{aligned}
\dot{V}(t) \leq & x^{T}(t)\left(P_{2}^{T} A+A^{T} P_{2}+Q_{1}+Q_{2}+Q_{3}+d_{2} W_{1}+\left(d_{2}-d_{1}\right) Z_{1}-\frac{1}{d_{2}} W_{3}+\beta_{1}^{2} \varepsilon_{1} I\right) x(t) \\
& +x^{T}(t)\left(P_{1}-P_{2}^{T}+A^{T} P_{3}+d_{2} W_{2}+\left(d_{2}-d_{1}\right) Z_{2}\right) y(t) \\
& +x^{T}(t)\left(-\frac{1}{d_{2}} W_{2}^{T}\right) \int_{t-d_{2}}^{t} x(s) d s+x^{T}(t)\left(-\frac{1}{d_{2}} W_{3}\right) x\left(t-d_{2}\right) \\
& +x^{T}(t)\left(P_{2}^{T} B\right) x(t-d(t))+x^{T}(t)\left(P_{2}^{T} C\right) y(t-d(t)) \\
& +x^{T}(t)\left(P_{2}^{T} G_{1}\right) f_{1}(t, x(t))+x^{T}(t)\left(P_{2}^{T} G_{2}\right) f_{2}(t, y(t)) \\
& +x^{T}(t)\left(P_{2}^{T} G_{3}\right) f_{3}(t, x(t-d(t)))+x^{T}(t)\left(P_{2}^{T} G_{4}\right) f_{4}(t, y(t-d(t))) \\
& +y^{T}(t)\left(2 P_{3}+d_{2} W_{3}+\left(d_{2}-d_{1}\right) Z_{3}+R+\beta_{2}^{2} \varepsilon_{2} I\right) y(t)+y^{T}(t)\left(P_{3} B\right) x(t-d(t)) \\
& +y^{T}(t)\left(P_{3} C\right) y(t-d(t))+y^{T}(t)\left(P_{3} G_{1}\right) f_{1}(t, x(t))+y^{T}(t)\left(P_{3} G_{2}\right) f_{2}(t, y(t)) \\
& +y^{T}(t)\left(P_{3} G_{3}\right) f_{3}(t, x(t-d(t)))+y^{T}(t)\left(P_{3} G_{4}\right) f_{4}(t, y(t-d(t))) \\
& +x^{T}\left(t-d_{1}\right)\left(-Q_{1}-\frac{1}{d_{2}-d_{1}} Z_{3}\right) x\left(t-d_{1}\right)+x^{T}\left(t-d_{1}\right)\left(\frac{1}{d_{2}-d_{1}} Z_{3}\right) x\left(t-d_{2}\right) \\
& +\xi^{T}(t) \Pi \xi(t) . \\
& +x_{t-d_{2}}^{T}\left(t-d_{1}\right)\left(-\frac{1}{d_{2}-d_{1}} Z_{2}^{T}\right) \int_{t-d_{2}}^{t-d_{1}} x(s) d s \\
& +x^{T}\left(t-d_{2}\right)\left(-Q_{2}-\frac{1}{d_{2}-d_{1}} Z_{3}-\frac{1}{d_{2}} W_{3}\right) x\left(t-d_{2}\right) \\
& +\int_{t-d_{2}}^{t} x^{T}(s) d s\left(-\frac{1}{d_{2}} W_{1}\right) \int_{t-d_{2}}^{t} x(s) d s \\
& +x^{T}\left(t-d_{2}\right)\left(\frac{1}{d_{2}} W_{2}^{T}\right) \int_{t-d_{2}}^{t} x(s) d s+x^{T}\left(t-d_{2}\right)\left(\frac{1}{d_{2}-d_{1}} Z_{2}^{T}\right) \int_{t-d_{2}}^{t-d_{1}} x(s) d s \\
& +x_{1}^{T}(t-d(t))\left(-Q_{3}+\beta_{3}^{2} \varepsilon_{3} I\right) x(t-d(t)) \\
& +y^{T}(t-d(t))\left(-(1-\bar{d}) R+\beta_{4}^{2} \varepsilon_{4} I\right) y(t-d(t)) \\
& +f_{1}^{T}(t, x(t))\left(-\varepsilon_{1} I\right) f_{1}(t, x(t))+f_{2}^{T}(t, y(t))\left(-\varepsilon_{2} I\right) f_{2}(t, y(t)) \\
& f_{4}^{T}(t, y(t-d(t)))\left(-\varepsilon_{3} I\right) f_{3}(t, x(t-d(t))) \\
& \\
& \\
&
\end{aligned}
$$

Define the extended vector

$$
\begin{aligned}
\xi(t)= & {\left[x^{T}(t), y^{T}(t), x^{T}\left(t-d_{1}\right), x^{T}\left(t-d_{2}\right), x^{T}(t-d(t)), y^{T}(t-d(t)), f_{1}^{T}(t, x(t)),\right.} \\
& \left.f_{2}^{T}(t, y(t)), f_{3}^{T}(t, x(t-d(t))), f_{4}^{T}(t, y(t-d(t))), \int_{t-d_{2}}^{t} x(s) d s, \int_{t-d_{2}}^{t-d_{1}} x(s) d s\right]^{T} .
\end{aligned}
$$

If $\Pi<0$, that is to say, $\dot{V}(t)<0$, then based on the Lyapunov method, system (2) is asymptotically stable, that is to say, system (1) is asymptotically stable. 
Furthermore, we prove the exponential stability of the neutral system (1).

Now, it is easy to see from (4) that there exists a scalar $\lambda_{0}>0$ such that for any $t, \dot{V}(t) \leq$ $-\lambda_{0}\|\Phi\|_{d}^{2}$.

Moreover, by the definition of a Lyapunov functional, there exist positive scalars $\lambda_{1}, \lambda_{2}$, $\lambda_{3}$, and $\lambda_{4}$ such that for any $t$ we have the following inequality:

$$
\begin{aligned}
V(t) \leq & \lambda_{1}\|x(t)\|^{2}+\lambda_{2} \int_{t-d_{2}}^{t}\|x(s)\|^{2} d s \\
& +\lambda_{3} \int_{t-d_{2}}^{t}\|\dot{x}(s)\|^{2} d s+\lambda_{4} \int_{t-d_{2}}^{t}\left\|x\left(s-d_{2}\right)\right\|^{2} d s .
\end{aligned}
$$

To prove the exponential stability of system (2), we define a new function by $W(t)=$ $e^{\varepsilon t} V(t)$, where the scalar $\varepsilon>0$. Then, we find that for any $t$,

$$
\begin{aligned}
W(t)-W(0)= & e^{\varepsilon t} V(t)-V(0) \\
= & \int_{0}^{t}\left[e^{\varepsilon s} \dot{V}(s)+\varepsilon e^{\varepsilon s} V(s)\right] d s \leq \int_{t}^{d} e^{\varepsilon s}[\varepsilon V(s)-\dot{V}(s)] d s \\
\leq & \int_{0}^{t} e^{\varepsilon \theta}\left[\varepsilon \lambda_{1}\|x(\theta)\|^{2}+\varepsilon \lambda_{2} \int_{\theta-d_{2}}^{\theta}\|x(s)\|^{2} d s+\varepsilon \lambda_{3} \int_{\theta-d_{2}}^{\theta}\|\dot{x}(s)\|^{2} d s\right. \\
& \left.+\varepsilon \lambda_{4} \int_{\theta-d_{2}}^{\theta}\left\|x\left(s-d_{2}\right)\right\|^{2} d s-\lambda_{0}\|\Phi\|_{d}^{2}\right] d \theta .
\end{aligned}
$$

By interchanging the integration sequence, we get

$$
\begin{aligned}
& \int_{0}^{t} e^{\varepsilon \theta} d \theta \int_{\theta-d_{2}}^{\theta}\|x(s)\|^{2} d s \leq d_{2} e^{\varepsilon d_{2}} \int_{0}^{t} e^{\varepsilon \theta}\|x(\theta)\|^{2} d \theta+d_{2}^{2} e^{\varepsilon d_{2}}\|\varphi\|^{2}, \\
& \int_{0}^{t} e^{\varepsilon \theta} d \theta \int_{\theta-d_{2}}^{\theta}\|\dot{x}(s)\|^{2} d s \leq d_{2} e^{\varepsilon d_{2}} \int_{0}^{t} e^{\varepsilon \theta}\|\dot{x}(\theta)\|^{2} d \theta+d_{2}^{2} e^{\varepsilon d_{2}}\|\phi\|^{2}, \\
& \int_{0}^{t} e^{\varepsilon \theta} d \theta \int_{\theta-d_{2}}^{\theta}\left\|x\left(s-d_{2}\right)\right\|^{2} d s \leq d_{2} e^{\varepsilon d_{2}} \int_{0}^{t} e^{\varepsilon \theta}\|x(\theta)\|^{2} d \theta+d_{2}^{2} e^{\varepsilon d_{2}}\|\varphi\|^{2} .
\end{aligned}
$$

Let $\varepsilon>0$ be small enough such that $\varepsilon \lambda_{1}+\varepsilon d_{2} e^{\varepsilon d_{2}} \lambda_{2}+\varepsilon d_{2} e^{\varepsilon d_{2}} \lambda_{3}-\lambda_{0} \leq 0$.

Then, substituting (9) into (8) shows that there exists a scalar $\beta>0$ such that, for any $t$,

$$
V(t) \leq \beta e^{-\varepsilon t}\|\Phi\|_{d}^{2}
$$

It can be seen that

$$
V(t) \geq\left[\begin{array}{l}
x(t) \\
y(t)
\end{array}\right]^{T}\left[\begin{array}{cc}
I_{r} & 0 \\
0 & 0
\end{array}\right]\left[\begin{array}{cc}
P_{1} & 0 \\
P_{2} & P_{3}
\end{array}\right]\left[\begin{array}{l}
x(t) \\
y(t)
\end{array}\right]=x^{T}(t) P_{1} x(t) \geq \lambda_{\min }\left(P_{1}\right)\|x(t)\|^{2} .
$$

Substituting (11) into (10) yields $\|x(t)\| \leq \sqrt{\frac{\beta}{\lambda_{\min \left(P_{1}\right)}}} e^{-\varepsilon t}\|\Phi\|_{d}$ for any $t$.

From Definition 1, $x(t)$ has exponential stability, that is, system (1) has exponential stability. This completes the proof. 
Remark 2 When $G_{1}=G_{2}=G_{3}=G_{4}=0$, system (1) is reduced to the traditional neutral system with time delay. However, for the class of neutral systems one has achieved some results in $[2,3]$ and the references therein.

Remark 3 When $G_{2}=0$ and $G_{1}=G_{3}=G_{4}=I$, system (1) is converted into the system in Ali [16]. However, our system has more the nature of universality.

Remark 4 When $C=0$ and $G_{4}=0$, system (1) reduces to the traditional power system with nonlinear perturbations (12). However, for the general power system with nonlinear perturbations one has achieved some results in $[9,16-18,20,21]$. We have

$$
\left\{\begin{array}{l}
\dot{x}(t)=A x(t)+B x(t-d(t))+G_{1} f_{1}(t, x(t))+G_{2} f_{2}(t, \dot{x}(t))+G_{3} f_{3}(t, x(t-d(t))), \\
x(t)=\phi(t), \quad \dot{x}(t)=\varphi(t), \quad t \in\left[-d_{2}, 0\right] .
\end{array}\right.
$$

Corollary 1 For prescribed scalar $d_{2}>0$, system (12) is exponentially admissible with convergence rate $\varepsilon$, if there exist positive-definite matrices $P=\left[\begin{array}{ll}P_{1} & 0 \\ P_{2} & P_{3}\end{array}\right]$ and positive-definite symmetric matrices $Q_{1}, Q_{2}, Q_{3}, W=\left[\begin{array}{cc}W_{1} & W_{2} \\ * & W_{3}\end{array}\right]$ and a real matrix $Z=\left[\begin{array}{cc}Z_{1} & Z_{2} \\ * & Z_{3}\end{array}\right]$ satisfying the following LMI:

$$
\left[\begin{array}{cccccccccc}
X_{11} & X_{12} & 0 & X_{14} & P_{2}^{T} B & P_{2}^{T} G_{1} & P_{2}^{T} G_{2} & P_{2}^{T} G_{3} & X_{1,9} & 0 \\
* & X_{22} & 0 & 0 & P_{3} B & P_{3} G_{1} & P_{3} G_{2} & P_{3} G_{3} & 0 & 0 \\
* & * & X_{33} & X_{34} & 0 & 0 & 0 & 0 & 0 & X_{3,10} \\
* & * & * & X_{44} & 0 & 0 & 0 & 0 & X_{4,9} & X_{4,10} \\
* & * & * & * & X_{55} & 0 & 0 & 0 & 0 & 0 \\
* & * & * & * & * & -\varepsilon_{1} I & 0 & 0 & 0 & 0 \\
* & * & * & * & * & * & -\varepsilon_{2} I & 0 & 0 & 0 \\
* & * & * & * & * & * & * & -\varepsilon_{3} I & 0 & 0 \\
* & * & * & * & * & * & * & * & X_{9,9} & 0 \\
* & * & * & * & * & * & * & * & * & X_{10,10}
\end{array}\right]<0,
$$

where

$$
\begin{aligned}
& X_{11}=P_{2}^{T} A+A^{T} P_{2}+Q_{1}+Q_{2}+Q_{3}+d_{2} W_{1}+\left(d_{2}-d_{1}\right) Z_{1}-\frac{1}{d_{2}} W_{3}+\beta_{1}^{2} \varepsilon_{1} I, \\
& X_{12}=P_{1}-P_{2}^{T}+A^{T} P_{3}+d_{2} W_{2}+\left(d_{2}-d_{1}\right) Z_{2}, \\
& X_{14}=-\frac{1}{d_{2}} W_{3}, \quad X_{1,9}=-\frac{1}{d_{2}} W_{2}^{T}, \\
& X_{22}=2 P_{3}+d_{2} W_{3}+\left(d_{2}-d_{1}\right) Z_{3}+\beta_{2}^{2} \varepsilon_{2} I, \quad X_{34}=\frac{1}{d_{2}-d_{1}} Z_{3}, \\
& X_{33}=-Q_{1}-\frac{1}{d_{2}-d_{1}} Z_{3}, \quad X_{3,10}=-\frac{1}{d_{2}-d_{1}} Z_{2}^{T}, \quad X_{4,9}=\frac{1}{d_{2}} W_{2}^{T}, \\
& X_{44}=-Q_{2}-\frac{1}{d_{2}-d_{1}} Z_{3}-\frac{1}{d_{2}} W_{3}, \quad X_{4,10}=\frac{1}{d_{2}-d_{1}} Z_{2}^{T}, \\
& X_{55}=-Q_{3}+\beta_{3}^{2} \varepsilon_{3} I, \quad X_{9,9}=-\frac{1}{d_{2}} W_{1}, \quad X_{10,10}=-\frac{1}{d_{2}-d_{1}} Z_{1} .
\end{aligned}
$$


Table 1 Maximum allowable time-delay upper bound for $\tau(t)=h(t)$

\begin{tabular}{ll}
\hline Result & $\begin{array}{l}\text { Upper bounds } \\
\text { of delays }\end{array}$ \\
\hline$[1]$ & 1.3955 \\
{$[2]$} & 0.7400 \\
{$[16]$} & 1.6100 \\
{$[19]$} & 1.7200 \\
{$[17]$} & 2.6000 \\
Ours & 3.1500 \\
\hline
\end{tabular}

Table 2 Maximum allowable time-delay upper bound for $d_{2}$

\begin{tabular}{lllllll}
\hline Time-delay conditions & {$[$ [2] } & {$[\mathbf{2 1 ]}$} & {$[$ 17] } & {$[18]$} & {$[16]$} & Ours \\
\hline$\alpha=0, \beta=0.1, \bar{d}=0$ & 0.6811 & 1.3279 & 2.7420 & 3.7440 & $d_{2}>0$ & $d_{2}>0$ \\
$\alpha=0, \beta=0.1, \bar{d}=0.5$ & 0.5467 & 0.6743 & 1.1420 & 1.4710 & 1.5774 & 1.7426 \\
$\alpha=0.1, \beta=0.1, \bar{d}=0$ & 0.6129 & 1.2503 & 1.8750 & 2.4430 & $d_{2}>0$ & $d_{2}>0$ \\
$\alpha=0.1, \beta=0.1, \bar{d}=0.5$ & 0.4950 & 0.5716 & 1.0090 & 1.2990 & 1.4196 & 2.3259 \\
\hline
\end{tabular}

\section{Numerical examples}

Example 1 Consider the following neutral time-delay system [16]:

$$
\begin{aligned}
\dot{x}(t)= & A x(t)+B x(t-d(t))+C \dot{x}(t-d(t))+G_{1} f_{1}(t, x(t))+G_{2} f_{2}(t, \dot{x}(t)) \\
& +G_{3} f_{3}(t, x(t-d(t)))+G_{4} f_{4}(t, \dot{x}(t-d(t))),
\end{aligned}
$$

with $A=\left[\begin{array}{cc}-2 & 0 \\ 0 & -2\end{array}\right], B=\left[\begin{array}{cc}0 & 0.4 \\ 0.4 & 0\end{array}\right], C=\left[\begin{array}{cc}0.1 & 0 \\ 0 & 0.1\end{array}\right], G_{1}=G_{3}=G_{4}=I$,

$$
G_{2}=0, \quad \alpha_{1}=0.1, \quad \alpha_{2}=0, \quad \alpha_{3}=0.05, \quad \dot{d}(t) \leq \bar{d}=0.5
$$

Using the Matlab LMI Toolbox and Theorem 1, we obtain maximum allowable upper bounds of $d_{2}$, which are listed in Table 1 . Table 1 describes maximum allowable upper bounds of delays that guarantee the exponential stability of system (1). It can be seen that our stability condition is less conservative than the results discussed in $[16,22,23]$.

Example 2 Consider the asymptotic stability with $\varepsilon=0$ of a system with time delay and nonlinear uncertainties in $[16-18,20,21]$ :

$$
\dot{x}(t)=A x(t)+B x(t-d(t))+G_{1} f_{1}(t, x(t))+G_{2} f_{2}(t, \dot{x}(t))+G_{3} f_{3}(t, x(t-d(t))),
$$

where

$$
A=\left[\begin{array}{cc}
-1.2 & 0.1 \\
-0.1 & -1
\end{array}\right], \quad B=\left[\begin{array}{cc}
-0.6 & 0.7 \\
-1 & -0.8
\end{array}\right] \text {. }
$$

By applying our criteria and using the Matlab LMI Toolbox, we have the comparative result listed in Table 2. From Table 2, we can see that our results are much less conservative than those in $[16-18,20,21]$.

\section{Conclusion}

The exponentially stability of a neutral system with nonlinear perturbations has been solved in terms of the LMI approach. Using the Lyapunov-Krasovskii functional method, 
the model transformation technique, and a new integral inequality, a new criterion for the exponential stability of systems is given. The criterion is presented in terms of linear matrix inequalities, which can easily be solved by the Matlab Toolbox and will have wide application in practical engineering. Finally, numerical examples are presented to illustrate the effectiveness of the method.

\section{Competing interests}

The authors declare that they have no competing interests.

\section{Authors' contributions}

LZ carried out the main part of this manuscript. YM participated in the discussion and corrected the main theorem. All authors read and approved the final manuscript.

\section{Acknowledgements}

This paper is supported by the National Natural Science Foundation of China (No. 61273004). The authors also gratefully acknowledge the helpful comments and suggestions of the reviewers, which have improved the presentation.

Received: 13 October 2013 Accepted: 8 January 2014 Published: 29 Jan 2014

\section{References}

1. Han, Q: On stability of linear neutral systems with mixed time delays: a discretized Lyapunov functional approach. Automatica 41, 1209-1218 (2005)

2. Han, Q: A descriptor system approach to robust stability of uncertain neutral systems with discrete and distributed delays. Automatica 49, 1791-1796 (2004)

3. Yue, D, Won, S, Kwon, O: Delay dependent stability of neutral systems with time-delay: an LMI approach. IEE Proc., Control Theory Appl. 150, 23-27 (2003)

4. Han, Q: A new delay-dependent absolute stability criterion for a class of nonlinear neutral systems. Automatica 44, 272-277 (2008)

5. Zhao, Z, Wang, W, Yang, B: Delay and its time-derivative dependent robust stability of neutral control system. Appl. Math. Comput. 187, 1326-1332 (2007)

6. Han, Q: On robust stability of neutral systems with time-varying discrete delay and norm-bounded uncertainty. Automatica 40, 1087-1092 (2004)

7. Zhu, S, Li, Z, Zhang, C: Delay decomposition approach to delay-dependent stability for singular time-delay systems. IET Control Theory Appl. 4, 2613-2620 (2010)

8. Park, JH: Novel robust stability criterion for a class of neutral systems with mixed delays and nonlinear perturbations. Appl. Math. Comput. 161, 413-421 (2005)

9. Zou, Z, Wang, Y: New stability criterion for a class of linear systems with time-varying delay and nonlinear perturbations. IEE Proc., Control Theory Appl. 153, 623-626 (2006)

10. Zhang, J, Peng, S, Ji, Q: Robust stability criterion for uncertain neutral system with time delay and nonlinear uncertainties. Chaos Solitons Fractals 38, 160-167 (2008)

11. Kwon, OM, Park, JH: Exponential stability for time-delay systems with interval time-varying delays and nonlinear perturbations. J. Optim. Theory Appl. 139, 277-293 (2008)

12. Liu, PL: Exponential stability for linear time-delay systems with delay-dependence. J. Franklin Inst. 340, 481-488 (2003)

13. Kwon, OM, Park, JH: Exponential stability of uncertain dynamic systems including state delay. Appl. Math. Lett. 19 901-907 (2006)

14. Yu, KW, Lien, $\mathrm{CH}$ : Stability criteria for uncertain neutral systems with interval time-varying delays. Chaos Solitons Fractals 38, 650-657 (2008)

15. Chen, Y, Xue, A, Lu, R, Zhou, S: On robustly exponential stability of uncertain neutral systems with time-varying delays and nonlinear perturbations. Nonlinear Anal. 68, 2464-2470 (2008)

16. Ali, MS: On exponential stability of neutral delay differential system with nonlinear uncertainties. Commun. Nonlinear Sci. Numer. Simul. 17, 2595-2601 (2012)

17. Zhang, JH, Shi, P, Qiu, JQ: Robust stability criteria for uncertain neutral system with time delay and nonlinear uncertainties. Chaos Solitons Fractals 38, 160-167 (2008)

18. Shen, CC, Zhong, SM: New delay-dependent robust stability criterion for neutral system with time-varying delay and nonlinear uncertainties. Chaos Solitons Fractals 40, 2277-2285 (2009)

19. Singh, V: Robust stability of cell neural networks with delay: linear matrix inequality approach. IEE Proc., Control Theory Appl. 151, 125-129 (2004)

20. Han, Q: Robust stability for a class of linear systems with time-varying delay and nonlinear perturbations. Comput. Math. Appl. 47, 1201-1209 (2004)

21. Cao, YY, Lam, J: Computation of robust stability bounds for time-delay systems with nonlinear time-varying perturbation. Int. J. Syst. Sci. 31, 359-365 (2000)

22. Park, JH, Won, S: Stability of neutral delay-differential systems with nonlinear perturbations. Int. J. Syst. Sci. 31, 961-967 (2000)

23. Yue, D, Fang, J, Won, S: Delay-dependent exponential stability of a class of neutral systems with time delay and time-varying parameter uncertainties: an LMI approach. JSME Int. J. Ser. C 46, 245-251 (2003) 
10.1186/1687-1847-2014-44

Cite this article as: Ma and Zhu: New exponential stability criteria for neutral system with time-varying delay and nonlinear perturbations. Advances in Difference Equations 2014, 2014:44

Submit your manuscript to a SpringerOpen ${ }^{\circ}$ journal and benefit from:

- Convenient online submission

- Rigorous peer review

- Immediate publication on acceptance

Open access: articles freely available online

- High visibility within the field

- Retaining the copyright to your article

Submit your next manuscript at $\boldsymbol{s p r i n g e r o p e n . c o m ~}$ 